Instituto Internacional de Investigación y Desarrollo Tecnológico Educativo INDTEC, C.A.

DOI: https://doi.org/10.29394/Scientific.issn.2542-2987.2020.5.15.7.150-167

OAI-PMH: http://www.indteca.com/ojs/index.php/Revista Scientific/oai

Artículo Original / Original Article

\title{
Lineamientos orientadores de las TIC como recurso didáctico en la Educación Primaria
}

Autor: Jerardin del Carmen Ríos Anciani Universidad Nacional Experimental "Rafael María Baralt", UNERMB jerardinrios@gmail.com Zulia, Venezuela https://orcid.org/0000-0003-4926-6435

\section{Resumen}

El presente artículo muestra los resultados de una investigación sobre las Tecnologías de la información y la comunicación (TIC) como recurso didáctico en la Educación primaria del Municipio Santa Rita. El objetivo general fue formular lineamientos orientadores de las TIC, para los docentes objeto de estudio. El estudio se enmarca en la metodología cuantitativa, descriptivo y de campo basado en el paradigma positivista. La muestra fue de 51 docentes y 14 directivos. Se aplicó como instrumento un cuestionario de escala tipo Likert. Entre los resultados, se logró establecer que la formación del docente sobre las TIC como recurso didáctico se ubica en una categoría moderada, con una baja dispersión, es decir que la formación docente técnica y didáctica debe fortalecerse para que asuman efectivamente su rol y puedan mejorar su praxis pedagógica en función del uso de las TIC como recurso didáctico, ya que no ha sido asumida de manera efectiva. Se proponen una serie de lineamientos orientadores para el uso de las Tic por los docentes de educación primaria.

Palabras clave: tecnología; información; comunicación.

Cómo citar este artículo:

Ríos, J. (2020). Lineamientos orientadores de las TIC como recurso didáctico en la Educación Primaria. Revista Scientific, 5(15), 150-167, e-ISSN: 2542-2987. Recuperado de: https://doi.org/10.29394/Scientific.issn.2542-2987.2020.5.15.7.150-167

Fecha de Recepción:

06-09-2019
Fecha de Aceptación:

13-12-2019
Fecha de Publicación:

05-02-2020 


\title{
Guidelines for ICT as a teaching resource in Primary Education
}

\begin{abstract}
This article shows the results of an investigation on Information and Communication Technologies (ICT) as a teaching resource in Primary Education of the Santa Rita Municipality. The general objective was to formulate guidelines for ICT for teachers under study. The study is part of the quantitative, descriptive and field methodology based on the positivist paradigm. The sample was 51 teachers and 14 managers. A Likert scale questionnaire was applied as an instrument. Among the results, it was established that teacher training on ICT as a teaching resource is located in a moderate category, with a low dispersion, that is to say that technical and didactic teacher training should be strengthened so that they effectively assume their role and can improve its pedagogical practice based on the use of ICT as a teaching resource, since it has not been effectively assumed. A series of guidelines for the use of the Tic by primary school teachers are proposed.
\end{abstract}

Keywords: technology; information; communication.

Date Received:

06-09-2019
Date Acceptance:

13-12-2019
Date Publication:

05-02-2020 


\section{Introducción}

Las Tecnologías de Información y Comunicación (TIC) han provocado cambios trascendentales en los esquemas que el hombre como ser pensante ha venido construyendo a lo largo de la vida en sociedad. Hoy en día prácticamente no existe actividad alguna que no involucre el uso de las TIC, debido a su vertiginosa evolución ha modificado la forma en la que se adquieren y transmite el conocimiento, de allí que la educación como uno de los más significativos procesos de evolución ha sido influenciada por la inclusión progresiva de dichas tecnologías en el quehacer pedagógico.

Destaca González (2016): que "las TIC pueden ir más allá de ser herramientas tanto para la Información como para la Comunicación, constituyéndose en verdaderos elementos de apoyo en la formación educativa" (pág. 495); con el fin de coadyuvar al fortalecimiento de una educación de calidad.

En este contexto, los sistemas educativos a nivel mundial se enfrentan actualmente al desafío de utilizar las TIC para proporcionar a los estudiantes los conocimientos necesarios para su desenvolvimiento en el Siglo XXI.

En referencia a lo anterior, Delors (1996), sostiene que:

Los sistemas educativos deben responder a los múltiples retos que les lanza la sociedad de la información, en función siempre de un enriquecimiento continuo de los conocimientos y del ejercicio de una ciudadanía adaptada a las exigencias de nuestra época (pág. 32).

En el contexto educativo venezolano, las TIC poseen un marco constitucional y curricular donde se han venido desarrollando grandes cambios a nivel curricular, y de políticas educativas por parte del estado en el ámbito de las TIC, con el fin de garantizar el acceso de la tecnología a las comunidades, concretado en la creación de instalaciones que prestaran tales servicios, como por ejemplo: los Centros Bolivarianos de Informática y 
Telemática (CBIT), aulas virtuales, Infocentro, laboratorios de informática, y la puesta en marcha del Proyecto Canaima educativo, contando además con la facilidad de conexión a la internet consolidada en una plataforma tecnológica acorde para la implementación de estos proyectos a través del Satélite Simón Bolívar en el año 2008.

En este orden de ideas, podemos señalar, que la escuela como un espacio abierto a la transformación, debe proveer a los estudiantes una formación integral congruente con los avances y desarrollo tecnológico, lo cual supone la necesidad de desarrollar un proceso de aprendizaje totalmente diferente al tradicional, esto demanda una nueva configuración del acto didáctico. No obstante, a pesar de los esfuerzos realizados por el gobierno Nacional, no termina de concretarse el hecho de integrar las TIC como recurso didáctico en las escuelas.

A este respecto, señalan Viñals y Cuenca (2016a), que:

Existe un cierto temor ante el uso de las TIC e Internet y sus consecuencias. Además, los medios de comunicación no han contribuido a proyectar las ventajas de la red, por lo que, de entrada, parece haberse instalado una sensación de inseguridad que ha repercutido en el ámbito educativo formal (pág. 109).

Sin duda alguna, existe desconocimiento por parte de los profesionales docentes sobre las ventajas que proporcionan el uso de la tecnología en la praxis docente, aunado al esfuerzo adicional que genera el aprender a utilizar algún dispositivo tecnológico, el tiempo que emplearía en elaborar recursos propios digitales, y principalmente el cambio de su metodología.

A esta realidad no escapan las instituciones educativas de la Parroquia "José Cenobio Urribarri", del Municipio Santa Rita del Estado Zulia; donde se pudo percibir que los docentes no acostumbraban incluir en su planificación diaria el uso de las TIC, alegando que tienen poco conocimiento acerca del manejo de manera específica en el software, o las aplicaciones implícitas 
como multimedia, gráficos, internet entre otras, esto quizás por poseer actitudes que pueden ser consideradas como "resistencia al cambio".

En consecuencia, en dichas instituciones continúa el modelo tradicional del profesor cuya actividad se basa en la transmisión de información bien sea a través de libros o materiales elaborados por ellos mismos con la utilización, en la mayoría de los casos de la pizarra, carteles, láminas, tableros, fotocopias, libros, donde las estrategias de aprendizaje están constituidas por discusiones y conversaciones de grupo, observándose poco el uso de las TIC como recurso didáctico dentro del proceso de enseñanza, aun cuando estas herramientas están a su alcance, como las Canaimitas, un recurso tan valioso que al parecer no son tomadas en cuenta, por los maestro.

Asimismo, expresan Paredes, Guitert y Rubia (2015): que "la mayoría de nuestras aulas siguen siendo un lugar donde lo fundamental es escuchar hablar al profesor, su explicación del contenido y participar en una evaluación para comprobar que el alumnado es capaz de reproducirlo" (pág. 106); evidentemente dejando fuera el uso de la tecnología y estrategias basadas en las TIC. Aunado a esto, no reconocen ni poseen lineamientos orientadores para la implementación de actividades que incluyan el uso de las TIC, y al no aprovecharlo en su momento, el docente pareciera no asumir sus roles, producto tal vez de mantener una planificación conductista.

Esta situación es motivada por la falta de interés en la aplicación de las tecnologías, desaprovechando las ventajas que estas aportan al proceso de enseñanza, aunado a la escasa supervisión del personal directivo que influye en la continuidad de esta situación, lo que puede generar el que prevalezca una educación rígida, que en ocasiones se puede convertir en repetitiva, sin dinamismo y lo holístico no se conceptualice mediante la intervención del estudiante de manera abierta. Como consecuencia de ello, este escenario favorece la presencia de un docente sin visión, que no desarrolle capacidades que lo conduzcan a espacios abiertos, limitando sus habilidades para generar 
aprendizajes de manera innovadora, además puede estar infiriendo en su actualización como profesional de manera tal que no le permita concretar saberes renovados.

En relación anterior, un informe de la OECD (2015), citado por Area, Hernández y Sosa (2016), indican que:

Los directores escolares señalan que los principales obstáculos para su uso son la falta de suficientes ordenadores, limitada conexión a Internet y escasez de software apropiado. También que los profesores consideran la formación en TIC como la segunda o tercera prioridad (pág. 80).

De allí que, es evidente la imperiosa necesidad de incorporar el uso de las TIC como recurso didáctico en las instituciones educativas de educación primaria como un elemento valioso que coadyuva al mejoramiento de los procesos de enseñanza aprendizaje y el desempeño efectivo de los docentes en sus diferentes funciones

Por su parte, Viñals y Cuenca (2016b), afirman lo siguiente:

Los docentes se enfrentan al reto de adquirir unas competencias que les formen para poder ayudar al alumnado a desarrollar las competencias que necesitan: conocimientos, habilidades y actitudes precisas para alcanzar los objetivos que se exigen desde el propio currículo formal (competencia digital y aprender a aprender, entre otras) para lograr adaptarse a las exigencias del mercado laboral, y aún más importante si cabe, para poder descubrir sus verdaderas motivaciones, intereses e inquietudes (pág. 110).

En relación a lo anterior, el docente debe tener una buena disposición para asumir dichos retos, y actualizar sus conocimientos de manera regular, para de esta manera contribuir con prácticas educativas efectivas a la formación integral de los educandos, constituye además, un llamado a la reflexión del personal educativo, en pro de generar cambios que beneficien el proceso educativo. Por ende, se ofrecen lineamientos orientadores sobre el 
uso de las TIC como recurso didáctico, como un elemento innovador que favorecerá en gran medida su papel como facilitador del aprendizaje.

\subsection{Componentes de las TIC en la formación técnica}

Actualmente, los docentes deben asumir una serie de cambios y transformaciones que se han tenido desarrollando desde hace ya varios años. El rol del alumno ha dejado de ser el receptor y por ende el rol del docente ya no consiste en la mera transmisión de conocimientos. De acuerdo con Araujo (2016): "se trata de que el educador sea el motor generador de sus propios cambios e innovaciones educativas, mediante la reflexión crítica, reflexiva de sus actuaciones en colaboración con sus compañeros" (pág. 188); así pues, la aplicación de las TIC por parte del docente requiere de un compromiso de formación, en todo cuanto respecta a las innovaciones tecnológicas existentes para que no se quede en la obsolescencia.

De esta manera, se presentan indicadores los cuales pueden considerarse como los componentes que requiere un docente en el uso de las TIC, de manera tal que haga reconocimiento de las diversas técnicas en el aula para ponerlas en práctica:

- Visión Compartida de los objetivos grupales.

- Capacitación para el desarrollo de elementos conceptuales, procedimentales y actitudinales.

- Disposición para realizar cambios en la metodología.

- Actualización de conocimientos que involucren el uso de las TIC.

- Uso de herramientas TIC: ofimáticas, programas, ocio, software, etc.

\subsection{Formación didáctica necesaria para el docente}

Cuando se relaciona la didáctica con la tecnología, se plantea entonces la capacidad que tiene el docente de facilitar el aprendizaje en los estudiantes, mediante la integración de recursos didácticos en el aula que permitan la 
asimilación de contenidos y construcción de saberes.

Cabe destacar, como afirma Cuevas y García (2014): "hay consenso en que la incorporación de las TIC en los procesos de formación de los docentes resulta indispensable para acometer los desafíos de la sociedad del conocimiento" (pág. 5). Los docentes han de adquirir habilidades como usuarios de los recursos digitales, no solo el mero conocimiento teórico o práctico del manejo de los recursos en forma aislada sino la forma en la que pedagógicamente estos recursos se emplean en el ámbito educativo, para así garantizar su integración de manera fluida tal como utiliza cualquier otro recurso como por ejemplo la pizarra, o las láminas.

En este sentido, como parte de la formación didáctica del docente, se debe tomar en cuenta los siguientes elementos en la formación didáctica:

- Interculturalidad, tomando en cuenta los elementos de la comunicación y diversidad en la cultura estudiantil.

- Relación teoría-practica, se refiere a los aspectos teóricos que son llevados a la práctica, aprender a aprender y aprender a hacer.

- Coherencia didáctica, tomando en consideración los elementos TIC, como recurso didáctico en el escenario de la educación primaria.

Ahora bien, el presente artículo proporciona un análisis crítico de la situación actual que se vive en las instituciones de educación primaria, en relación a la formación escasa del docente para manejar, usar e implementar recursos Tic en su quehacer diario. Por ende, la relevancia de este estudio radica en la necesidad de implementar una metodología activa, en las instituciones educativas del municipio Santa Rita, a través del seguimiento de una serie de lineamientos que orienten sus prácticas educativas en pro de mejorar la efectividad en el desempeño de sus funciones y por ende la calidad educativa.

En este sentido, el objetivo consistió en formular lineamientos 
orientadores de las Tecnologías de Información y Comunicación como recurso didáctico en los docentes de las escuelas de la Parroquia José Cenobio Urribarrí, Municipio Santa Rita del Estado Zulia, Venezuela.

\section{Metodología}

Con el fin de alcanzar el objetivo propuesto fue necesario efectuar una revisión de la literatura relacionada con las variables de estudio y definir los aspectos metodológicos concernientes a la investigación llevada a cabo. En este sentido, se realizaron una serie de visitas a las instituciones educativas pertenecientes a la parroquia José Cenobio Urribarrí, del Municipio Santa Rita, estado Zulia, Venezuela con la finalidad de observar la situación de los docentes al momento de utilizar las TIC como recurso didáctico en el aula de clases. La población estuvo constituida por 65 personas, 51 docentes y 14 directivos.

Para la recolección de datos, se diseñaron dos cuestionarios, identificado como: (C-ID1) para los docentes y (C-ID2) para los directivos, de escala tipo Likert, con las alternativas de respuesta (5: Siempre; 4: Casi siempre; 3: A veces; 2: Nunca; 1: Casi Nunca), con el fin de recabar la información necesaria en la investigación, dicho instrumento fue validado mediante juicio de 3 expertos en el área, utilizando una guía de validación.

La validación interna se realizó a través de la aplicación del método Alpha Cronbach para doble alternativa, en una hoja de cálculo del programa Excel obteniendo un coeficiente de $84 \%$, lo cual indica que el instrumento mide confiablemente la variable de estudio. Asimismo, para calcular la confiabilidad de los cuestionarios, se utilizó el Método de Estadística de las dos mitades (pares e impares), en hoja de cálculo con la corrección de Spearman Brown, arrojando como resultado $99 \%$, lo que indica que el instrumento posee un alto grado de confiabilidad. 


\section{Análisis e Interpretación de Resultados}

Las reflexiones y análisis efectuados, son producto de la información recabada mediante la aplicación de los cuestionarios (C-ID1) para los docentes y (C-ID2) para los directivos, en relación a la variable de estudio denominada: Tecnologías de información y comunicación y recurso didáctico, de acuerdo a sus dimensiones e indicadores, cuyas categorías fueron analizadas de acuerdo a la interpretación del promedio; clasificándose de acuerdo a los intervalos establecidos en: muy alta (4.21-5), alta (3.41-4.20), moderada (2.61-3.40), baja (1.81-2.60) y muy baja (1-1.80). Por su parte, para la interpretación de la desviación estándar, se tomó como referencia los valores: muy alta Dispersión (3.21-4), alta dispersión (2.41-3.20), moderada dispersión (1.61-2.40), baja dispersión (0.81-1.60), y muy baja dispersión (0$0.80)$.

El procedimiento realizado para la interpretación de los resultados consistió en totalizar los puntajes obtenidos en las alternativas de respuesta descritas anteriormente, por cada ítems, dentro de las categorías, y posteriormente agruparlos por dimensión expresados en porcentajes. A continuación, se muestran los resultados obtenidos para la dimensión Formación técnica de los docentes, en la tabla 1.

Tabla 1. Componentes de las TIC's en la formación técnica de los docentes.

\begin{tabular}{lcccc}
\hline \multirow{2}{*}{ Indicadores } & \multicolumn{2}{c}{ Directiva } & \multicolumn{2}{c}{ Docentes } \\
\cline { 2 - 5 } & Promedio & Desviación & Promedio & Desviación \\
\hline Visión Compartida & 3,03 & 0,20 & 3,08 & 0,27 \\
\hline Capacitación & 3,01 & 0,27 & 3,04 & 0,21 \\
\hline Disposición & 3,00 & 0,18 & 3,05 & 0,22 \\
\hline Actualización & 3.00 & 0,22 & 3.06 & 0,22 \\
\hline Aplicación & 3.00 & 0,22 & 3.06 & 0,20 \\
\hline Promedio General & $\mathbf{3 . 0 0}$ & $\mathbf{0 , 2 2}$ & $\mathbf{3 , 0 6}$ & $\mathbf{0 , 2 3}$ \\
\hline
\end{tabular}

Fuente: La Autora (2019).

De acuerdo a las respuestas suministradas por los encuestados, fue 
posible la interpretación de los resultados, basados en la información teórica recopilada y enriquecido con la opinión de la investigadora con el propósito de dar respuesta al objetivo y generar el nuevo conocimiento en relación a la temática de estudio.

En lo referido a la formación técnica en TIC, se evidencia, para la directiva, un promedio en sus respuestas de 3,00 , con una muy baja dispersión de 0.22; ubicándose dentro de la categoría moderada; afirmando los encuestados, las carencias que se presentan en cuanto al desarrollar una visión compartida, y amplia disposición, que conlleve a ellos como directiva y a los docentes a actualizarse para saber aplicar las TIC.

En cuanto a la opinión de los docentes, se evidencia un promedio en sus respuestas de 3.06 , con una muy baja dispersión de 0.23 , ubicándose en una categoría moderada; afirmando, que si bien desarrollan aspectos en la formación técnica para la utilización de las Tic como docentes, tendrían una percepción más amplia a través de sus actuaciones, ya sea dentro del aula o fuera de ella donde la directiva juega desde lo académico un gran papel. En relación a la dimensión de formación didáctica del docente los resultados para la variable se muestran en la tabla 2.

Tabla 2. Formación didáctica necesaria para el docente.

\begin{tabular}{lllll}
\hline \multirow{2}{*}{\multicolumn{1}{c}{ Indicadores }} & \multicolumn{2}{c}{ Directiva } & \multicolumn{2}{c}{ Docentes } \\
\cline { 2 - 5 } & Promedio & Desviación & Promedio & Desviación \\
\hline Interculturalidad & 3.41 & 0,24 & 3.06 & 0,20 \\
\hline Relación Teoría-practica & 3.41 & 0,28 & 3.06 & 0,19 \\
\hline Coherencia didáctica & 3.41 & 0,19 & 3.06 & 0,19 \\
\hline Promedio General & $\mathbf{3 . 4 1}$ & $\mathbf{0 , 2 1}$ & $\mathbf{3 . 0 6}$ & $\mathbf{0 , 1 9}$
\end{tabular}

Fuente: La Autora (2019).

En relación a la formación didáctica necesaria para el docente, se evidenció para el directivo un promedio de 3.41 , categoría alta con una muy baja dispersión de 0.21 , esto permite considerar que tanto en la institución y la 
gestión que realizan existe la disposición en cuanto a las TIC y una coherencia didáctica consciente, asimismo la interculturalidad se maneja de manera asertiva.

No obstante, sobre la base de las respuestas alcanzadas a través de encuestas realizadas a los docentes, se obtuvo un promedio de 3,06 con una muy baja dispersión de 0.19 , ubicándose en una categoría moderada, lo que permite inferir que estos asumen la relevancia de las TIC, en la formación didáctica puesto que la practican, pero muchos de ellos no la relacionan con la tecnología dentro del aula, por ende, la teoría y práctica se ven afectados. Por su parte, la interculturalidad, aunque asertiva debe ser más puntualizada.

Por consiguiente, producto de la investigación es necesario proponer lineamientos orientadores del uso y aplicación de las Tecnologías de Información y Comunicación como recurso didáctico en los docentes de las escuelas de la Parroquia José Cenobio Urribarrí, Municipio Santa Rita del Estado Zulia, para lo cual se tiene lo siguiente:

- El proceso de enseñanza aprendizaje, es un proceso cocreador, en el cual tanto el educador, como el educando aprenden simultáneamente y se enriquecen desarrollando al máximo las potencialidades. Dentro de este proceso constructivo del conocimiento entran en juego el uso de recursos didácticos que promuevan y fomenten aprendizajes innovadores y faciliten a su vez la adquisición del conocimiento, así como también la labor del maestro en facilitarla.

- Dentro de este contexto, el uso de la tecnología en el aula funciona como apoyo didáctico al docente, impulsando estrategias y actividades que integran lo visual, auditivo, interactivo, multimedia e imágenes, incentiva la investigación, el trabajo colaborativo entre estudiantesestudiantes, docentes-estudiantes, docentes-docentes, inclusive favorece la denominada triada: escuela-familia y comunidad. 
- El uso de las TIC, constituyen hoy en día, un pilar fundamental para la construcción del conocimiento, tanto dentro como fuera del aula, su uso, e integración va más allá de lo que puede hacer o crear el maestro dentro del salón de clases. Sin duda alguna, se han convertido en una herramienta de cambios, en tal sentido, la gestión educativa además de asumirlas ha de propiciar encuentros para que sus instituciones se vinculen, diligenciando la dotación de esta herramienta. Se hace necesario entonces, que este componente lo internalice para establecer los parámetros que en la práctica lo justifiquen.

- En base a lo expresado; contando las instituciones con las herramientas implícitas en las TIC, (centro de informática), se hace necesario la presencia de expertos que técnicamente generen competencias al personal directivo, así como a los docentes en el manejo de los equipos de informática, y asimismo la comunidad pueda integrarse de manera efectiva en las actividades vinculadas con la tecnología en la institución.

- Es necesario crear en la comunidad educativa redes o espacios para compartir experiencias relacionadas al uso de las TIC en el aula. En este particular, el estado venezolano en los últimos años promueve congresos pedagógicos a nivel estatal, local y nacional, donde se analizan y discuten diferentes problemáticas educativas, es importante destacar la relevancia de las TIC, así como la necesidad de formación docente que garantice las buenas prácticas educativas basadas en el uso de la tecnología como un recurso didáctico que añade a la labor docente dinamismo y actualidad.

- Se destaca que, dentro de las directrices emanadas por el gobierno nacional, en el Plan Nacional Simón Bolívar en los periodos 2019-2025, se enfatizan lo concerniente a la tecnología través de sus objetivos, considerando: El fomentar la ciencia y la tecnología al servicio del 
pueblo con el fin de generar la justa distribución de la tecnología entre las comunidades y garantizar a través de ellas el desarrollo de la nación, por medio del contexto socio educativo, y socio cultural.

- En este contexto, la educación tecnológica juega una función hacia la formación de los protagonistas de las instituciones, se habla de la directiva, personal administrativo y los docentes, quienes a su vez sumaran información y formación a los estudiantes, por ser el eje principal del quehacer curricular la cual marca la innovación mediante el uso de recursos didácticos.

- El paradigma de las nuevas tecnologías está representado por el uso de la Internet, es necesario realizar dentro de la gestión directiva las diligencias pertinentes que garanticen a la comunidad educativa el acceso a dicho recurso con el fin de mejorar el acceso a la información y crear diferentes recursos educativos, a la vez de actividades didácticas basadas en la web tales como; investigación en internet, difusión de actividades y relación con otras instituciones educativas, mediante blogs, sitios web, redes sociales, además de tener a disponibilidad una gama de recursos on-line para el trabajo con los alumnos, como software educativos, y diversas aplicaciones informáticas.

- Considerando lo manifestado, se hace imprescindible generar los mecanismos de aprendizajes (formación técnica) del personal docente para que comience a dejar de resistirse, entendiendo con ello que como formador está en la obligación de transformar los aprendizajes, donde las TIC, brinda oportunidades para ilustrar pedagógicamente los nuevos conocimientos de los estudiantes.

- Es necesario la transformación del perfil de competencias del personal docente y directivo de las instituciones educación primaria, tomando 
como referencia la formación técnica y didáctica tomando en cuenta las nuevas exigencias de la sociedad actual y la realidad tecnológica de su país, para de esta manera actuar con coherencia en pro de mejorar la calidad de los procesos educativos, llevados a cabo en la institución.

- El docente, asumiendo su rol innovador, debe estar a la aplicación de herramientas didácticas novedosas; las cuales en estos tiempos modernos permite la introducción de la tecnología. En este sentido, es necesario una comprensión y entendimiento sobre la relevancia de las TIC en el ejercicio docente, es necesario organizar seminarios, talleres, jornadas y congresos sobre el uso de la tecnología; en función de crear nuevos saberes y nuevos paradigmas para hacer de la enseñanza un proceso constructivo de innovación, dinamismo, interactividad, reflexión y autoaprendizaje.

- En la medida que el docente reciba y acepte la formación de nuevos conocimientos técnicos, y didácticos, entenderá que los centros de informática presentes en sus instituciones, son un recurso, que al ser usados con inteligencia; podrán favorecerla atención, retención de los nuevos conocimientos además aprender jugando, a través de las animaciones, el internet y otros elementos implícitos en las TIC.

\section{Conclusiones}

Luego de haber realizado el análisis correspondiente al estudio se concluye en referencia al objetivo general sobre la variable: Tecnologías de Información y comunicación como recursos didáctico tanto el personal directivo como docente, en su nivel de conocimiento sobre las TIC aún no termina de fortalecerse, de lo que puede deducirse la necesidad de un mayor desarrollo en la formación técnica y didáctica del docente, y es necesario que la directiva, impulse mecanismos para elevar sus conocimientos teóricos - 
prácticos en el área, ya que se observó resistencia por parte de los mismos.

Igualmente, la directiva debe asumir el compromiso de seguir contribuyendo a la formación personal y profesional del personal a su cargo para obtener mayores y mejores beneficios, conscientes de las necesidades reales que presenta su equipo de trabajo y quienes son los principales agentes de cambio dentro y fuera de la organización, por ende, la reflexión en este punto es crucial para determinar cambios profundos a nivel gerencial.

Los hallazgos encontrados en la investigación permiten analizar la formación presente en los docentes objeto del estudio y su percepción ante el uso de las TIC, los resultados obtenidos pueden servir de referencia para futuras investigaciones relacionadas, y la metodología utilizada también puede aplicarse en casos similares que tengan que ver con la formación técnica y didáctica del docente en las dimensiones caracterizadas en este artículo.

Se proponen entonces una serie de lineamientos orientadores de las Tic como recurso didáctico en los docentes de las escuelas de la Parroquia José Cenobio Urribarrí, Municipio Santa Rita del Estado Zulia, como producto de la investigadora en función de los conocimientos adquiridos durante el proceso investigativo.

\section{Referencias}

Araujo, M. (2016). Formación del Docente de Educación Media General desde el Enfoque por Competencias. Revista Scientific, 1(2), 181199, e-ISSN: 2542-2987. Recuperado de:

https://doi.org/10.29394/scientific.issn.2542-2987.2016.1.2.11.181-199

Area, M., Hernández, V., \& Sosa, J. (2016). Modelos de integración didáctica de las TIC en el aula. Comunicar, XXIV(47), 79-87, ISSN: 1134-3478; e-ISSN: 1988-3293. Recuperado de:

https://doi.org/10.3916/C47-2016-08

Cuevas, F., \& García, J. (2014). Las TIC en la formación docente. Artículo 
1159. Congreso Iberoamericano de Ciencia, Tecnología, Innovación y Educación. ISBN: 978-84-7666-210-6. Buenos Aires, Argentina: Organización de Estados Iberoamericanos (OEI). Recuperado de: https://www.oei.es/historico/congreso2014/memoriactei/1159.pdf Delors, J. (1996). La educación encierra un tesoro. Informe a la UNESCO de la Comisión Internacional sobre la Educación para el siglo XXI, presenciada por Jacques Delors. ISBN: 92-3-303274-4. París, Francia: Santillana - Ediciones UNESCO. Recuperado de: http://www.unesco.org/education/pdf/DELORS S.PDF

González, M. (2016). Formación docente en competencias tic para la mediación de aprendizajes en el proyecto Canaima Educativo. Telos, 18(3), 492-507, e-ISSN: 1317-0570. Recuperado de: http://www.redalyc.org/articulo.oa?id=99346931009

Paredes, J., Guitert, M., \& Rubia, B. (2015). La innovación y la tecnología educativa como base de la formación inicial del profesorado para la renovación de la enseñanza. RELATEC. Revista Latinoamericana de Tecnología Educativa, 14(1), 101-114, e-ISSN: 1695-288X. Recuperado de: http://doi.org/10.17398/1695-288X.14.1.101

Viñals, A., \& Cuenca, J. (2016a,b). El rol del docente en la era digital. Revista Interuniversitaria de Formación del Profesorado, 30(2), 103-114, eISSN: 0213-8646. Recuperado de:

http://www.redalyc.org/articulo.oa?id=27447325008 


\section{Jerardin del Carmen Ríos Anciani}

e-mail: jerardinrios@gmail.com

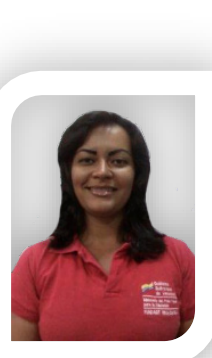

Nacida en Cabimas estado Zulia, Venezuela, el 4 de febrero del año 1985. Obtuve el título de Bachiller en Ciencias en la Unidad Educativa José Isidro Silva; realicé estudios de pregrado en Universidad Nacional Experimental "Rafael María Baralt" (UNERMB), obteniendo el título de Licenciada en Educación Integral en el año 2007; donde también obtuve el título de Magister Scientiarum en Administración de la Educación en el año 2015; graduada como T.S.U. en Informática de la Misión Sucre en la Universidad Bolivariana de Venezuela (UBV, 2019); Desempeño funciones como Docente de Recursos para el Aprendizaje desde el año 2008 hasta la actualidad.

El contenido de este manuscrito se difunde bajo una Licencia de Creative Commons ReconocimientoNoComercial-Compartirlgual 4.0 Internacional 